PROCEEDINGS OF THE

AMERICAN MATHEMATICAL SOCIETY

Volume 139, Number 8, August 2011, Pages 2877-2889

S 0002-9939(2011)11034-7

Article electronically published on March 29, 2011

\title{
INNER FUNCTIONS AND SPHERICAL ISOMETRIES
}

\author{
MICHAEL DIDAS AND JÖRG ESCHMEIER
}

(Communicated by Richard Rochberg)

\begin{abstract}
A commuting tuple $T=\left(T_{1}, \ldots, T_{n}\right) \in B(H)^{n}$ of bounded Hilbert-space operators is called a spherical isometry if $\sum_{i=1}^{n} T_{i}^{*} T_{i}=1_{H}$. B. Prunaru initiated the study of $T$-Toeplitz operators, which he defined to be the solutions $X \in B(H)$ of the fixed-point equation $\sum_{i=1}^{n} T_{i}^{*} X T_{i}=X$. Using results of Aleksandrov on abstract inner functions, we show that $X \in B(H)$ is a $T$-Toeplitz operator precisely when $X$ satisfies $J^{*} X J=X$ for every isometry $J$ in the unital dual algebra $\mathcal{A}_{T} \subset B(H)$ generated by $T$. As a consequence we deduce that a spherical isometry $T$ has empty point spectrum if and only if the only compact $T$-Toeplitz operator is the zero operator. Moreover, we show that if $\sigma_{p}(T)=\emptyset$, then an operator which commutes modulo the finite-rank operators with $\mathcal{A}_{T}$ is a finite-rank perturbation of a $T$-Toeplitz operator.
\end{abstract}

\section{INTRODUCTION}

Let $H$ denote a separable complex Hilbert space. A spherical isometry is a commuting tuple $T=\left(T_{1}, \ldots, T_{n}\right) \in B(H)^{n}$ of continuous linear operators on $H$ satisfying

$$
T_{1}^{*} T_{1}+T_{2}^{*} T_{2}+\cdots+T_{n}^{*} T_{n}=1_{H}
$$

Since this condition is modelled after the defining function for the boundary $\partial \mathbb{B}_{n}$ of the Euclidean unit ball $\mathbb{B}_{n}$ in $\mathbb{C}^{n}$, one should expect that the operator theory of such a tuple $T$ is closely related to the function theory on $\mathbb{B}_{n}$. The desired link is given by a result of Athavale (Proposition 2 in [2]) saying that each spherical isometry is subnormal. More explicitly, for each spherical isometry $T \in B(H)^{n}$ there exist a Hilbert space $K \supset H$ and a spherical unitary, that is, a commuting tuple $U \in B(K)^{n}$ of normal operators with Taylor spectrum $\sigma(U) \subset \partial \mathbb{B}_{n}$, such that $T_{i}=U_{i} \mid H$ for $i=1, \ldots, n$. Replacing $K$ by $\bigvee_{\alpha \in \mathbb{N}_{0}^{n}}\left(U^{*}\right)^{\alpha} H$, if necessary, we may assume that $U$ is the minimal normal extension of $T$, which is known to be unique up to unitary equivalence.

Let $E(\cdot)$ denote the projection-valued spectral measure for $U$. If we fix a separating unit vector $z \in H$ for $W^{*}(U)$, then the probability measure $\mu=\langle E(\cdot) z, z\rangle$ is a scalar-valued spectral measure of $U$. Since $\mu$ is supported by the Taylor spectrum of $U$, we may regard $\mu$ as a measure on $\partial \mathbb{B}_{n}$. This measure will be referred to in the sequel as the scalar-valued spectral measure associated with $T$. Note that $\mu$ is unique modulo mutually absolute continuity. By the spectral theorem for

Received by the editors July 28, 2010.

2010 Mathematics Subject Classification. Primary 47A13, 47B20, 47L45; Secondary 47B35, $47 \mathrm{~L} 80$.

(C)2011 American Mathematical Society 
commuting tuples of normal operators, there is an isomorphism of von Neumann algebras

$$
\Psi_{U}: L^{\infty}(\mu) \rightarrow W^{*}(U) \subset B(K)
$$

mapping the coordinate functions $z_{i}$ to the operators $U_{i}(i=1, \ldots, n)$. If we define $H^{\infty}(\mu)$ to be the dual subalgebra

$$
H^{\infty}(\mu)={\overline{\left\{\left[p \mid \partial \mathbb{B}_{n}\right]_{\mu}: p \in \mathbb{C}[z]\right\}}}^{w^{*}} \subset L^{\infty}(\mu)
$$

and write

$$
\mathcal{A}_{T}=\overline{\{p(T): p \in \mathbb{C}[z]\}}^{w^{*}} \subset B(H)
$$

for the unital dual operator algebra generated by $T$, then it is well known that the map $\Psi_{U}$ induces a dual algebra isomorphism, that is, an isometric isomorphism and weak* homeomorphism,

$$
\gamma_{T}: H^{\infty}(\mu) \rightarrow \mathcal{A}_{T}, \quad f \mapsto \Psi_{U}(f) \mid H
$$

which extends the polynomial functional calculus of $T$ in a unique way (see Conway 6. Proposition 1.1). It is an elementary and well-known fact that this isomorphism yields a correspondence between $\mu$-inner functions, that is, functions

$$
\theta \in H^{\infty}(\mu) \text { with }|\theta|=1 \quad \mu \text {-almost everywhere on } \partial \mathbb{B}_{n},
$$

and isometries in the dual algebra generated by $T$. For completeness sake, we give a proof.

Lemma 1.1. Let $T \in B(H)^{n}$ be a spherical isometry with associated spectral measure $\mu \in M^{+}\left(\partial \mathbb{B}_{n}\right)$. An operator $J \in \mathcal{A}_{T}$ is an isometry if and only if $J=\gamma_{T}(\theta)$ with some $\mu$-inner function $\theta \in H^{\infty}(\mu)$.

Proof. For every $x \in H$ and every $\theta \in H^{\infty}(\mu)$, we have

$$
\left\|\gamma_{T}(\theta) x\right\|^{2}=\left\|\Psi_{U}(\theta) x\right\|^{2}=\left\langle\Psi_{U}\left(|\theta|^{2}\right) x, x\right\rangle
$$

If $\theta$ is inner, then this observation implies that $\gamma_{T}(\theta)$ is an isometry. For the reverse direction, remember that $\mu=\langle E(\cdot) z, z\rangle$ with a separating vector $z \in H$ for $U$. So if $J \in \mathcal{A}_{T}$ is assumed to be an isometry and $J=\gamma_{T}(\theta)$ with $\theta \in H^{\infty}(\mu)$, then we obtain by the above equality applied to $x=z$ that

$$
0=\|z\|^{2}-\left\langle\Psi_{U}\left(|\theta|^{2}\right) z, z\right\rangle=\left\langle\Psi_{U}\left(1-|\theta|^{2}\right) z, z\right\rangle=\int_{\partial \mathbb{B}_{n}}\left(1-|\theta|^{2}\right) d \mu .
$$

The identity $\|\theta\|_{\infty, \mu}=\|J\|=1$ shows that the integrand $1-|\theta|^{2}$ is non-negative and hence equal to zero $\mu$-almost everywhere.

Via this correspondence, richness and approximation results for $\mu$-inner functions immediately yield information about the structure of the dual algebra $\mathcal{A}_{T}$. The aim of the next section is to collect and extend some known approximation results for $\mu$-inner functions. 


\section{Abstract INNER FUnCTIONS}

The results in this section will be formulated in a more general setting than necessary for the study of spherical isometries. In the sequel we replace the unit sphere $\partial \mathbb{B}_{n}$ by a general compact subset $K \subset \mathbb{C}^{n}$. We write $M^{+}(K)$ for the set of all positive regular Borel measures on $K$ and $C(K)$ for the space of all $\mathbb{C}$-valued continuous functions on $K$.

Given a unital closed subalgebra $A \subset C(K)$ containing the polynomials $\mathbb{C}[z] \mid K$ in $n$ complex variables $z=\left(z_{1}, \ldots, z_{n}\right)$ and a measure $\mu \in M^{+}(K)$, the triple $(A, K, \mu)$ is called regular in the sense of Aleksandrov [1] if, for every function $\varphi \in C(K)$ with $\varphi>0$, there exists a sequence $\left(f_{k}\right)$ in $A$ with $\left|f_{k}\right|<\varphi$ for all $k$ and $\lim _{k \rightarrow \infty}\left|f_{k}\right|=\varphi \mu$-almost everywhere on $K$. Obviously, if $\nu \in M^{+}(K)$ is absolutely continuous with respect to $\mu$ and $(A, K, \mu)$ is regular, then so is $(A, K, \nu)$. For a regular triple $(A, K, \mu)$, the support of $\mu$ is necessarily contained in the Shilov boundary $S(A)$ of $A$. Remember that $S(A)$ is the smallest closed set $S \subset K$ with the property that $\|f\|_{\infty, K}=\|f\|_{\infty, S}$ for every $f \in A$.

To give an example of a regular triple relevant for spherical isometries, let

$$
A\left(\mathbb{B}_{n}\right)=\left\{f \in C\left(\overline{\mathbb{B}}_{n}\right): f \mid \mathbb{B}_{n} \text { is holomorphic }\right\} \subset C\left(\overline{\mathbb{B}}_{n}\right)
$$

be the ball algebra. For $K=\partial \mathbb{B}_{n}$ and $A=A\left(\mathbb{B}_{n}\right) \mid K$, which is isomorphic to $A\left(\mathbb{B}_{n}\right)$ by the maximum-modulus principle, Aleksandrov showed in [1] that the triple $(A, K, \mu)$ is regular for every regular Borel measure $\mu \in M^{+}\left(\partial \mathbb{B}_{n}\right)$.

Before we can formulate Aleksandrov's existence result for inner functions with respect to regular triples, we have to gather some more notation. Recall that a measure $\mu \in M^{+}(K)$ is said to be continuous if $\mu(\{\zeta\})=0$ for every $\zeta \in K$, and discrete if there is a countable set $\Delta \subset K$ with $\mu(K \backslash \Delta)=0$. Note that for each measure $\mu \in M^{+}(K)$, the set $\Delta=\{\zeta \in K: \mu(\{\zeta\})>0\}$ is countable and $\mu$ possesses a decomposition $\mu=\mu_{d}+\mu_{c}$ into a discrete and a continuous part defined by

$$
\mu_{d}(\omega)=\mu(\omega \cap \Delta) \quad \text { and } \quad \mu_{c}(\omega)=\mu(\omega \cap(K \backslash \Delta))
$$

for every Borel set $\omega \subset K$. The elements of $\Delta$ are called (one-point) atoms of $\mu$.

In the sequel, we write $H_{A}^{\infty}(\mu)$ for the weak* closure of $A$ in $L^{\infty}(\mu)$ and $H_{A}^{2}(\mu)$ for the norm closure of $A$ in $L^{2}(\mu)$. We write

$$
I_{\mu}=\left\{\theta \in H_{A}^{\infty}(\mu):|\theta|=1 \quad \mu \text {-almost everywhere }\right\} \subset L^{\infty}(\mu)
$$

for the set of $\mu$-inner functions. The following approximation theorem (see Corollary 29 in [1]) shows that for a regular triple based on a continuous measure, the algebra $H_{A}^{\infty}(\mu)$ has a rich supply of inner functions.

Proposition 2.1 (Aleksandrov). Let $\mu \in M^{+}(K)$ be a continuous measure such that the triple $(A, K, \mu)$ is regular. Then the weak $*^{*}$ closure of the set

$$
I_{\mu}=\left\{\theta \in H_{A}^{\infty}(\mu):|\theta|=1 \quad \mu \text {-almost everywhere }\right\}
$$

contains the $L^{\infty}(\mu)$-equivalence classes of all functions $f \in A$ with $|f| \leq 1$ on $K$. In particular, $H_{A}^{\infty}(\mu)=\overline{\mathrm{LH}}^{w^{*}}\left(I_{\mu}\right)$ is the weak $k^{*}$ closed linear span of $I_{\mu}$.

Since $L^{1}(\mu)$ is separable, the weak* topology on the closed unit ball $B$ of $L^{\infty}(\mu)$ is metrizable. Since $I_{\mu} \subset B$, the weak* closure and the weak* sequential closure of $I_{\mu}$ in $L^{\infty}(\mu)$ coincide. Thus, for every $f \in A$ with $\|f\|_{\infty, K} \leq 1$, there is a sequence of inner functions $\theta_{k} \in I_{\mu}\left(k \in \mathbb{N}_{0}\right)$ with weak ${ }^{*}$ limit $f$. 
Let $1 \leq p \leq \infty$ and let $\mu=\mu_{d}+\mu_{c}$ be the decomposition of a given measure $\mu \in M^{+}(K)$ into its discrete and continuous part. Then there is a natural isometric isomorphism $\sigma_{p}: L^{p}(\mu) \longrightarrow L^{p}\left(\mu_{d}\right) \oplus_{p} L^{p}\left(\mu_{c}\right)$,

$$
[f]_{\mu} \mapsto\left([f]_{\mu_{d}},[f]_{\mu_{c}}\right)=\left(\left[f \chi_{\Delta}\right]_{\mu_{d}},\left[f \chi_{K \backslash \Delta}\right]_{\mu_{c}}\right)
$$

with inverse given by $\sigma_{p}^{-1}\left([g]_{\mu_{d}},[h]_{\mu_{c}}\right)=\left[g \chi_{\Delta}+h \chi_{K \backslash \Delta}\right]_{\mu}$. Note that in the case $p=\infty$, the map $\sigma_{\infty}: L^{\infty}(\mu) \rightarrow L^{\infty}\left(\mu_{d}\right) \oplus_{\infty} L^{\infty}\left(\mu_{c}\right)$ is not only an isometric isomorphism but also (as the adjoint of $\sigma_{1}^{-1}$ ) a weak ${ }^{*}$ homeomorphism.

In the case of a regular triple $(A, K, \mu)$, the above isomorphisms lead to natural direct sum decompositions of $H_{A}^{p}(\mu)$. The proof will be based on the following observation.

Lemma 2.2. Let $(A, K, \mu)$ be a regular triple, and let $\{\zeta\} \subset K$ be a one-point atom of $\mu$. Then, for each $\epsilon>0$, there exist an open neighborhood $U$ of $\zeta$ with $\mu(U \backslash\{\zeta\})<\epsilon$ and a function $g \in A$ with $|g| \leq 1$ on $K$ and

$$
|g(\zeta)-1|<\epsilon, \quad|g|<\epsilon \quad \text { on } K \backslash U .
$$

Proof. Choose an open neighborhood $U$ of $\zeta$ with $\mu(U \backslash\{\zeta\})<\epsilon$. By Tietze's extension theorem there is a positive continuous function $\varphi: K \rightarrow[\epsilon, 1]$ with $\varphi(\zeta)=1$ and $\varphi \mid K \backslash U=\epsilon$. Since $(A, K, \mu)$ is regular, there exists a sequence of functions $\left(g_{j}\right)$ in $A$ such that $\left|g_{j}\right|<\varphi$ on $K$ and $\left|g_{j}\right| \stackrel{j}{\rightarrow} \varphi \mu$-almost everywhere on $K$. Since $\zeta$ has positive $\mu$-measure, this implies that $\left|g_{j}(\zeta)\right| \rightarrow 1$ as $j \rightarrow \infty$. Therefore the function $g=\alpha g_{k}$, where $k \in \mathbb{N}$ is sufficiently large and $\alpha \in \mathbb{C}$ is a suitably chosen complex number with $|\alpha|=1$, has all the desired properties.

As a consequence we obtain the following decomposition result.

Proposition 2.3. For a regular triple $(A, K, \mu)$, the mappings $\sigma_{p}(1 \leq p \leq \infty)$ defined above induce isomorphisms

$$
H_{A}^{p}(\mu) \cong L^{p}\left(\mu_{d}\right) \oplus_{p} H_{A}^{p}\left(\mu_{c}\right) .
$$

Proof. Fix an arbitrary point $\zeta \in \Delta$ and use Lemma 2.2 with $\epsilon=1 / k$ to choose a sequence of functions $g_{k} \in A$ with the properties stated there. It is not hard to show (cf. Lemma 3 in [11] for the $H^{2}$-case) that the sequence $\left(g_{k}\right)$ converges to $\chi_{\{\zeta\}}$ in the $L^{p}(\mu)$-norm for $1 \leq p<\infty$ and in the weak* topology of $L^{\infty}(\mu)$ for $p=\infty$. This observation implies that the right-hand side is contained in the image of $H_{A}^{p}(\mu)$ under $\sigma_{p}$. The reverse inclusion is obvious.

In the setting of Proposition 2.3 we find in particular that $L^{p}\left(\mu_{d}\right)=H_{A}^{p}\left(\mu_{d}\right)$ for $1 \leq p \leq \infty$. The decomposition obtained for $H_{A}^{\infty}(\mu)$ allows us to prove an approximation result similar to Proposition 2.1 without any continuity assumption on the underlying measure. Though weaker than the original one, this observation still has some interesting applications in operator theory. Below we shall use the following notation. Given arbitrary elements $\delta \in L^{\infty}\left(\mu_{d}\right)$ and $\theta \in L^{\infty}\left(\mu_{c}\right)$, we write $\delta \oplus \theta$ for the element $\sigma_{\infty}^{-1}(\delta, \theta) \in L^{\infty}(\mu)$. To be more specific, if $\delta=[g]_{\mu_{d}}$ and $\theta=[h]_{\mu_{c}}$ with representatives $g, h: K \rightarrow \mathbb{C}$, then

$$
\delta \oplus \theta=\sigma_{\infty}^{-1}(\delta, \theta)=\left[g \chi_{\Delta}+h \chi_{K \backslash \Delta}\right]_{\mu} \in L^{\infty}(\mu) .
$$

Note that $\delta \oplus \theta$ is a $\mu$-inner function whenever $\delta$ is a $\mu_{d}$-inner function and $\theta$ is a $\mu_{c}$-inner function.

Now we are ready for the proof of the announced density result. 
Proposition 2.4. For every regular triple $(A, K, \mu)$, without any continuity assumption on $\mu$, we have that

$$
H_{A}^{\infty}(\mu)=\overline{\mathrm{LH}}^{w^{*}}\left(I_{\mu}\right)
$$

Proof. It suffices to show that every $f \in A$ with $\|f\|_{\infty, K} \leq 1$ belongs to the weak* closure on the right-hand side. The direct sum representation $H_{A}^{\infty}(\mu) \cong$ $L^{\infty}\left(\mu_{d}\right) \oplus_{\infty} H_{A}^{\infty}\left(\mu_{c}\right)$ allows us to decompose $[f]_{\mu}=[g]_{\mu_{d}} \oplus[h]_{\mu_{c}}$ with $g=f \chi_{\Delta}$ and $h=f \chi_{K \backslash \Delta}$, where $\Delta$ denotes the set of all one-point atoms of $\mu$. By the cited result of Aleksandrov (see Proposition 2.1 and the subsequent remark), the continuous part $[h]_{\mu_{c}}$ can be approximated in the weak* topology of $L^{\infty}\left(\mu_{c}\right)$ by a sequence $\left(\theta_{k}\right)_{k \geq 0}$ of $\mu_{c}$-inner functions $\theta_{k} \in I_{\mu_{c}} \subset H_{A}^{\infty}\left(\mu_{c}\right)$.

To treat the discrete part, observe that $|\Re g|,|\Im g| \leq|f| \leq 1$. So we may define four bounded measurable functions $u_{+}, u_{-}, v_{+}, v_{-}: K \rightarrow \mathbb{C}$ by the formulas

$$
u_{ \pm}=\Re g \pm i \sqrt{1-(\Re g)^{2}}, \quad v_{ \pm}=\Im g \pm i \sqrt{1-(\Im g)^{2}} .
$$

If $u$ is any of these four functions $u_{ \pm}, v_{ \pm}$, then by construction $|u|=1$ on $K$. Since $L^{\infty}\left(\mu_{d}\right)=H_{A}^{\infty}\left(\mu_{d}\right)$, the equivalence classes $\delta_{ \pm}=\left[u_{ \pm}\right]_{\mu_{d}}$ and $\rho_{ \pm}=\left[v_{ \pm}\right]_{\mu_{d}}$ are $\mu_{d}$-inner functions. Furthermore, $[g]_{\mu_{d}}=\frac{1}{2}\left(\delta_{+}+\delta_{-}\right)+\frac{i}{2}\left(\rho_{+}+\rho_{-}\right)$.

Now we can solve the approximation problem for the initial function $f \in A$. Having in mind the remarks preceding the proposition, we define four sequences of inner functions in $H_{A}^{\infty}(\mu)$ by setting

$$
\eta_{k}^{1}=\delta_{+} \oplus \theta_{k}, \quad \eta_{k}^{2}=\delta_{-} \oplus \theta_{k}, \quad \eta_{k}^{3}=\rho_{+} \oplus \theta_{k}, \quad \eta_{k}^{4}=\rho_{-} \oplus\left(-\theta_{k}\right)
$$

for $k \geq 0$. By construction, the sequence $\left(f_{k}\right)$ with

$$
f_{k}=\frac{1}{2}\left(\eta_{k}^{1}+\eta_{k}^{2}\right)+\frac{i}{2}\left(\eta_{k}^{3}+\eta_{k}^{4}\right) \quad(k \geq 0)
$$

belongs to $\operatorname{LH}\left(I_{\mu}\right)$ and has $f$ as its weak* limit, since $\sigma_{\infty}$ is a weak* homeomorphism.

Using the weak ${ }^{*}$ density of the polynomials in $z$ and $\bar{z}$ one obtains a corresponding density result for $L^{\infty}(\mu)$.

Corollary 2.5. In the situation of the preceding proposition, we also have

$$
L^{\infty}(\mu)=\overline{\mathrm{LH}}^{w^{*}}\left(\left\{\bar{\eta} \cdot \theta: \eta, \theta \in I_{\mu}\right\}\right) .
$$

Proof. Since the complex polynomials in $z$ and $\bar{z}$ are weak* dense in $L^{\infty}(\mu)$, it suffices to show that, for arbitrary multi-indices $\alpha, \beta \in \mathbb{N}_{0}^{n}$, the monomials $z^{\alpha} \bar{z}^{\beta}$ are in the weak* closure of the set $\operatorname{LH}\left(\left\{\bar{\eta} \cdot \theta: \eta, \theta \in I_{\mu}\right\}\right)$. According to the previous proposition and its proof, there exist sequences $\left(f_{k}\right)$ and $\left(g_{k}\right)$ in $\operatorname{LH}\left(I_{\mu}\right)$ with weak ${ }^{*}$ limits $z^{\alpha}$ and $z^{\beta}$, respectively. Then, for every $k \in \mathbb{N}$, the function $f_{k} \bar{z}^{\beta}=\mathrm{w}^{*}$ $\lim _{j \rightarrow \infty} f_{k} \overline{g_{j}}$ belongs to $\overline{\mathrm{LH}}^{w^{*}}\left(\left\{\bar{\eta} \cdot \theta: \eta, \theta \in I_{\mu}\right\}\right)$. Hence the same is true for the functions $z^{\alpha} \bar{z}^{\beta}=\mathrm{w}^{*}-\lim _{k \rightarrow \infty} f_{k} \bar{z}^{\beta}$.

\section{Spherical isometries and Toeplitz operators}

We apply the approximation results established in the preceding section to the particular case $K=\partial \mathbb{B}_{n}$ and $A=A\left(\mathbb{B}_{n}\right) \mid \partial \mathbb{B}_{n}$. Since the polynomials are norm dense in $A\left(\mathbb{B}_{n}\right)$, the dual algebra $H_{A}^{\infty}(\mu)$ coincides with the weak* closure $H^{\infty}(\mu)$ 
of the polynomials in $L^{\infty}(\mu)$. Let $T \in B(H)^{n}$ be a spherical isometry with scalarvalued spectral measure $\mu \in M^{+}\left(\partial \mathbb{B}_{n}\right)$ and let $U, \Psi_{U}, \gamma_{T}$ be defined as in the introduction. We denote by

$$
\mathcal{I}_{T}=\left\{J: J \text { is an isometry in } \mathcal{A}_{T}\right\}
$$

the set of all isometries $J \in B(H)$ belonging to the dual algebra $\mathcal{A}_{T}$ generated by the spherical isometry $T$.

A) Reflexivity revisited. With every subset $\mathcal{S} \subset B(H)$ one associates the WOTclosed unital operator algebra $\operatorname{AlgLat}(\mathcal{S})$ consisting of all operators $C$ in $B(H)$ that leave invariant every closed $\mathcal{S}$-invariant subspace of $H$. The family $\mathcal{S}$ is called reflexive if the identity

$$
\operatorname{AlgLat}(\mathcal{S})=\mathcal{W}_{\mathcal{S}}
$$

holds, where $\mathcal{W}_{\mathcal{S}}$ denotes the smallest WOT-closed subalgebra containing $1_{H}$ and $\mathcal{S}$. A commuting tuple $T \in B(H)^{n}$ is called reflexive if $\operatorname{Alg} \operatorname{Lat}(T)=\mathcal{W}_{T}$, where by a slight abuse of language the symbol $T$ refers to the subset $\left\{T_{1}, \ldots, T_{n}\right\} \subset B(H)$.

The fact that spherical isometries are reflexive has been shown by the first author in [9]. The proof given there can be shortened by using Proposition 2.4. Note that this proposition and Lemma 1.1 guarantee that

$$
\mathcal{A}_{T}=\overline{\mathrm{LH}}^{w^{*}}\left(\mathcal{I}_{T}\right) \text {. }
$$

As a family of commuting isometries, $\mathcal{I}_{T}$ is reflexive by a result of Bercovici (see Theorem 2.4 in [4]). Hence we obtain that

$$
\operatorname{AlgLat}(T)=\operatorname{AlgLat}\left(\mathcal{A}_{T}\right)=\operatorname{AlgLat}\left(\mathcal{I}_{T}\right)=\mathcal{W}_{\mathcal{I}_{T}} \subset \overline{\operatorname{ran}\left(\gamma_{T}\right)}{ }^{W O T}=\mathcal{W}_{T},
$$

without using a decomposition of $T$ into its unitary and pure part as in 9 .

B) Toeplitz operators. Recall that a classical result of Brown and Halmos says that an operator $X \in B\left(H^{2}(\mathbb{D})\right)$ is a Toeplitz operator with symbol in $L^{\infty}(\mathbb{T})$ if and only if $T^{*} X T=X$, where $T=M_{z}$ denotes the multiplication with $z$ which is an isometry on $H^{2}(\mathbb{D})$. Generalizing this algebraic condition, Prunaru [14] defines the set of all Toeplitz operators with respect to a spherical isometry $T \in B(H)^{n}$ as

$$
\mathcal{T}(T)=\left\{X \in B(H): \sum_{i=1}^{n} T_{i}^{*} X T_{i}=X\right\} .
$$

Prunaru even extends this definition to commuting families of spherical isometries. Given an arbitrary index set $\Gamma$ and natural numbers $n_{\alpha} \in \mathbb{N}(\alpha \in \Gamma)$, a family $\mathcal{F}=\left(T_{\alpha}\right)_{\alpha \in \Gamma}$ of multi-operators is called a commuting family of spherical isometries if each operator tuple $T_{\alpha}=\left(T_{\alpha, 1}, \ldots, T_{\alpha, n_{\alpha}}\right) \in B(H)^{n_{\alpha}}$ is a spherical isometry and all operators $T_{\alpha, j}$ with $\alpha \in \Gamma$ and $1 \leq j \leq n_{\alpha}$ commute with each other. Following 14] the set of all $\mathcal{F}$-Toeplitz operators is then defined to be

$$
\mathcal{T}(\mathcal{F})=\bigcap_{\alpha \in \Gamma} \mathcal{T}\left(T_{\alpha}\right)
$$

One of the main results of Prunaru (see part (3) of Theorem 1.2 in [14]) shows that

$$
\mathcal{T}(\mathcal{F})=\left\{P_{H} Y \mid H: Y \in(\widehat{\mathcal{F}})^{\prime}\right\},
$$

where $\widehat{\mathcal{F}}=\left(N_{\alpha}\right)$ is the minimal normal extension of the family $\mathcal{F}=\left(T_{\alpha}\right)$ and $(\widehat{\mathcal{F}})^{\prime}=\bigcap\left(\left(N_{\alpha, j}\right)^{\prime}: \alpha \in \Gamma, 1 \leq j \leq n_{\alpha}\right) \subset B(K)$ denotes the commutant of $\widehat{\mathcal{F}}$. 
Now let us return from this abstract setting to a concrete situation, namely the Hardy space $H=H^{2}\left(\mathbb{B}_{n}\right)$ over the unit ball in $\mathbb{C}^{n}$. Obviously the tuple $T=$ $\left(M_{z_{1}}, \ldots, M_{z_{n}}\right) \in B(H)^{n}$ of multiplication operators with the coordinate functions is a spherical isometry. Guo and Wang observed (Proposition 2.1 in [13) that the $T$-Toeplitz operators are characterized by the condition that $M_{\eta}^{*} X M_{\eta}=X$ for every inner function $\eta \in H^{\infty}\left(\mathbb{B}_{n}\right)$. The following is an abstract analogue of this result in the context of spherical isometries.

Proposition 3.1. Let $T \in B(H)^{n}$ be a spherical isometry. An operator $X \in B(H)$ is a T-Toeplitz operator, that is, satisfies the equation $\sum_{i=1}^{n} T_{i}^{*} X T_{i}=X$ if and only if

$$
J^{*} X J=X \quad \text { for every isometry } J \text { in the dual algebra } \mathcal{A}_{T} .
$$

Proof. With the notation as before consider the operator families $\mathcal{I}_{T}=\left(\gamma_{T}(\theta)\right)_{\theta \in I_{\mu}}$ on $H$ and $\mathcal{I}_{U}=\left(\Psi_{U}(\theta)\right)_{\theta \in I_{\mu}}$ on $K$. Note that $\mathcal{I}_{T}$ consists of commuting isometries and that $\mathcal{I}_{U}$ consists of commuting unitary operators. From the density result established in Corollary 2.5 we deduce that

$$
W^{*}(U)=\Psi_{U}\left(L^{\infty}(\mu)\right)=\overline{\mathrm{LH}}^{w^{*}}\left(\left\{A^{*} B: A, B \in \mathcal{I}_{U}\right\}\right) .
$$

This implies that every subspace $M \subset K$ which is reducing for $\mathcal{I}_{U}$ also reduces $U$. Hence $\mathcal{I}_{U}$ is the minimal normal extension of $\mathcal{I}_{T}$. Furthermore, Proposition 2.4 implies that

$$
\mathcal{A}_{U}=\Psi_{U}\left(H^{\infty}(\mu)\right)=\overline{\mathrm{LH}}^{w^{*}}\left(\mathcal{I}_{U}\right) .
$$

Hence the commutants $(U)^{\prime}=\left(\mathcal{A}_{U}\right)^{\prime}=\left(\mathcal{I}_{U}\right)^{\prime}$ coincide. By Theorem 1.2 in Prunaru [14] we obtain that

$$
\mathcal{T}(T)=P_{H}(U)^{\prime}\left|H=P_{H}\left(\mathcal{I}_{U}\right)^{\prime}\right| H=\mathcal{T}\left(\mathcal{I}_{T}\right) .
$$

Thus the proof is complete.

Our next aim is to show that the decomposition $\mu=\mu_{c}+\mu_{d}$ of the spectral measure $\mu \in M^{+}\left(\partial \mathbb{B}_{n}\right)$ associated with a spherical isometry $T \in B(H)^{n}$ into its continuous and discrete part gives rise to an orthogonal decomposition $H=H_{c} \oplus H_{d}$ which is reducing for every $T$-Toeplitz operator. Before proving this, we recall that $\mu(\{\zeta\})>0$ for some $\zeta \in \partial \mathbb{B}_{n}$ if and only if $\zeta$ is an eigenvalue of the minimal normal extension $U \in B(K)^{n}$ of $T$ and that in this case the operator $P_{\zeta}=\Psi_{U}\left(\chi_{\{\zeta\}}\right) \in B(K)$ is the orthogonal projection onto the joint eigenspace $\bigcap_{i=1}^{n} \operatorname{ker}\left(\zeta_{i}-U_{i}\right)$. As before, let $\Delta$ be the set of all one-point atoms of $\mu$. Fix a point $\zeta \in \Delta$. By Proposition 2.3 it follows that $\chi_{\{\zeta\}} \in H^{\infty}(\mu)$. Hence $H$ is invariant for the orthogonal projection $P_{\zeta}=\Psi_{U}\left(\chi_{\{\zeta\}}\right)$. Since for every vector $x \in P_{\zeta} H^{\perp} \subset H^{\perp}$, the space $(\mathbb{C} x)^{\perp}$ contains $H$ and is reducing for $U$, the minimality of $U$ implies that

$$
\bigcap_{i=1}^{n} \operatorname{ker}\left(\zeta_{i}-U_{i}\right)=P_{\zeta} K=P_{\zeta} H=\bigcap_{i=1}^{n} \operatorname{ker}\left(\zeta_{i}-T_{i}\right) .
$$

In particular, it follows that $\sigma_{p}(T)=\sigma_{p}(U)=\Delta$, where $\sigma_{p}$ stands for the point spectrum of the underlying tuple. In the sequel, we abbreviate by

$$
H_{d}^{\zeta}=\bigcap_{i=1}^{n} \operatorname{ker}\left(\zeta_{i}-T_{i}\right)=\bigcap_{i=1}^{n} \operatorname{ker}\left(\zeta_{i}-U_{i}\right) \quad\left(\zeta \in \sigma_{p}(T)\right)
$$


the corresponding eigenspaces and define $H_{d}=\bigoplus_{\zeta \in \sigma_{p}(T)} H_{d}^{\zeta}$, where the $d$ in the notation refers to the discrete part. The orthogonal complement of $H_{d}$ is denoted by $H_{c}=H \ominus H_{d}$.

A spherical isometry $T \in B(H)^{n}$ for which $H_{d}$ is zero will be called continuous. So $T$ is continuous if and only if $\mu$ is a continuous measure.

Proposition 3.2. Let $T \in B(H)^{n}$ be a spherical isometry. The orthogonal decomposition $H=H_{c} \oplus \bigoplus_{\zeta \in \sigma_{p}(T)} H_{d}^{\zeta}$ reduces all T-Toeplitz operators and gives rise to an $\ell^{\infty}$-direct sum decomposition

$$
\mathcal{T}(T) \cong \mathcal{T}\left(T_{c}\right) \oplus \bigoplus_{\zeta \in \sigma_{p}(T)} B\left(H_{d}^{\zeta}\right),
$$

where $T_{c}=T \mid H_{c} \in B\left(H_{c}\right)^{n}$ is a continuous spherical isometry.

Proof. Let $\zeta$ be an eigenvalue of $T$ or, equivalently, a one-point atom of $\mu$. As seen above, the projection $P_{\zeta}=\Psi_{U}\left(\chi_{\{\zeta\}}\right)$ leaves $H$ invariant and thus commutes with $P_{H} \in B(K)$. The restriction $Q_{\zeta}=P_{\zeta} \mid H$ is the orthogonal projection from $H$ onto $H_{d}^{\zeta}$. So given any $T$-Toeplitz operator $X=P_{H} A \mid H$ with $A \in(U)^{\prime}$ and an arbitrary vector $h \in H$ we find that

$$
X Q_{\zeta} h=P_{H}(A \mid H) Q_{\zeta} h=P_{H} A P_{\zeta} h=P_{\zeta} P_{H}(A \mid H) h=Q_{\zeta} X h .
$$

This proves the first part of the assertion.

Fix an operator $X_{c} \in \mathcal{T}\left(T_{c}\right)$ and a bounded family of operators $X_{\zeta} \in B\left(H_{d}^{\zeta}\right)$. Define $X=X_{c} \oplus \bigoplus_{\zeta} X_{\zeta} \in B(H)$. Given $h \in H$, we decompose $h=h_{c} \oplus \bigoplus_{\zeta} h_{\zeta}$ to obtain

$\sum_{i=1}^{n} T_{i}^{*} X T_{i} h=\sum_{i=1}^{n}\left(T_{c}\right)_{i}^{*} X_{c}\left(T_{c}\right)_{i} h_{c} \oplus \bigoplus_{\zeta \in \sigma_{p}(T)} \sum_{i=1}^{n} \overline{\zeta_{i}} X_{\zeta} \zeta_{i} h_{\zeta}=X_{c} h_{c} \oplus \bigoplus_{\zeta} X_{\zeta} h_{\zeta}=X h$.

Thus $X \in \mathcal{T}(T)$, and the proof is complete.

Let $\mathcal{K}(H) \subset B(H)$ denote the set of all compact operators on $H$. In the classical theory of Toeplitz operators on the Hardy space of the unit disc, Brown and Halmos observed in [5] that $X \in \mathcal{K}(H)$ is a Toeplitz operator if and only if $X=0$. The corresponding result in our abstract setting is the following.

Theorem 3.3. A spherical isometry $T \in B(H)^{n}$ has empty point spectrum if and only if the zero-operator is the only compact $T$-Toeplitz operator. In this case, we have

$$
\|X\|=\inf \{\|X-K\|: K \in \mathcal{K}(H)\}
$$

for all T-Toeplitz operators $X \in B(H)$.

Proof. Suppose that $\zeta \in \sigma_{p}(T)$ is an eigenvalue of $T$. Fix a rank-one operator $X_{\zeta}$ in $B\left(H_{d}^{\zeta}\right)$ on the corresponding eigenspace $H_{d}^{\zeta}$. In view of the preceding proposition we obtain a non-zero compact $T$-Toeplitz operator by setting $X h=X_{\zeta} P_{\zeta} h(h \in H)$.

On the other hand, if $\sigma_{p}(T)=\emptyset$, then $T=T_{c}$ and the spectral measure $\mu$ associated with $T$ is continuous. Thus there exists a weak* zero sequence $\left(\theta_{k}\right)_{k \geq 0}$ of $\mu$-inner functions by Aleksandrov's approximation theorem (Proposition 2.1). Since the functional calculus $\gamma_{T}: H^{\infty}(\mu) \rightarrow B(H)$ is weak* continuous, the corresponding sequence of isometries $J_{k}=\gamma_{T}\left(\theta_{k}\right) \in B(H)$ is a weak* zero sequence. Let $X \in \mathcal{T}(T)$ 
be a $T$-Toeplitz operator on $H$. Using Proposition 3.1 we obtain for each unit vector $h \in H$ and each compact operator $K \in \mathcal{K}(H)$ the estimates

$$
\|X-K\| \geq\left\|J_{k}^{*}(X-K) J_{k} h\right\|=\left\|X h-J_{k}^{*} K J_{k} h\right\| \quad(k \geq 0) .
$$

Since $\left(J_{k} h\right)$ is a weak zero sequence, the compactness of $K$ implies that $K J_{k} h \stackrel{k}{\longrightarrow} 0$ in norm. It follows that

$$
\|X-K\| \geq \lim _{k \rightarrow \infty}\left\|X h-J_{k}^{*} K J_{k} h\right\|=\|X h\| .
$$

Since this is true for every unit vector $h \in H$ and every compact operator $K$ in $K(H)$, we conclude that $\|X\|=\inf \{\|X-K\|: K \in \mathcal{K}(H)\}$. In particular, the zero operator is the only compact $T$-Toeplitz operator.

As another application of Proposition 3.1 we deduce a necessary condition for an operator $S \in B(H)$ to have finite-rank commutators

$$
[S, A]=S A-A S \quad \text { for every } \quad A \in \mathcal{A}_{T} .
$$

Questions like this are inspired by a work of Davidson [7], who succeeded in identifying the essential commutant of the set of all analytic Toeplitz operators on the Hardy space $H^{2}(\mathbb{D})$ over the unit disc. A generalization to the case of the unit ball has been established by Guo in [12. As a variation of this theme, Guo and Wang [13] characterized all operators $S \in B\left(H^{2}\left(\mathbb{B}_{n}\right)\right)$ having finite-rank commutators $\left[S, T_{f}\right]$ for all Toeplitz operators $T_{f} \in B\left(H^{2}\left(\mathbb{B}_{n}\right)\right)$ with bounded analytic symbol $f \in H^{\infty}\left(\mathbb{B}_{n}\right)$.

In the sequel we closely follow the work of Guo and Wang [13] and the corresponding ideas from Davidson 7 .

Lemma 3.4. (a) Let $\left(F_{k}\right)_{k \geq 1}$ be a sequence in $B(H)$ satisfying $\operatorname{rank}\left(F_{k}\right) \leq M$ for $k \geq 1$ with some fixed natural number $M$. If $\left(F_{k}\right)$ has a WOT-limit $F \in B(H)$, then $\operatorname{rank}(F) \leq M$.

(b) Let $\mathcal{A} \subset B(H)$ be a closed subspace. If $S \in B(H)$ has the property that $[S, A]$ is of finite rank for all $A \in \mathcal{A}$, then there exists a constant $M>0$ such that $\operatorname{rank}([S, A]) \leq M$ for all $A \in \mathcal{A}$.

Proof. The proof is almost a word-by-word repetition of that in [13]. For the reader's convenience, we state it here. Assuming $\operatorname{rank}(F)>M$ one can find $N=$ $M+1$ vectors $x_{1}, \ldots, x_{N} \in H$ and an orthonormal system $\left\{y_{1}, \ldots, y_{N}\right\} \subset H$ such that $d=\operatorname{det}\left(\left\langle F x_{i}, y_{j}\right\rangle_{i j}\right) \neq 0$. Since for every $k \in \mathbb{N}$ the set of vectors $\left\{F_{k} x_{1}, \ldots, F_{k} x_{N}\right\}$ is linearly dependent, we obtain $0=\operatorname{det}\left(\left\langle F_{k} x_{i}, y_{j}\right\rangle_{i j}\right) \stackrel{k \rightarrow \infty}{\longrightarrow} d \neq 0$, a contradiction which finishes the proof of part (a).

The hypothesis of part (b) guarantees that the Banach space $\mathcal{A}$ can be represented as the union $\mathcal{A}=\bigcup_{k=1}^{\infty} \Gamma_{k}$ of the norm-closed sets $\Gamma_{k}=\{A \in \mathcal{A}$ : $\operatorname{rank}([S, A]) \leq k\}$. Baire's category theorem implies the existence of an inner point $A_{0}$ in some $\Gamma_{N}$ with $N \in \mathbb{N}$. Since $\Gamma_{N}-A_{0}$ is an open neighborhood of zero in the Banach space $\mathcal{A}$, every operator $C \in \mathcal{A}$ satisfies $C / \alpha \in \Gamma_{N}-A_{0}$ with some suitably chosen number $\alpha=\alpha(C)>0$. Hence $C \in \alpha \Gamma_{N}-\alpha \Gamma_{N}$, implying that $\operatorname{rank}([S, C]) \leq 2 N$.

Theorem 3.1 in Guo-Wang [13] says that for $T=M_{z} \in B\left(H^{2}\left(\mathbb{B}_{n}\right)\right)^{n}$ and $n \geq 2$, an operator $S \in B\left(H^{2}\left(\mathbb{B}_{n}\right)\right)$ commutes modulo the finite-rank operators with all analytic Toeplitz operators $T_{f}\left(f \in H^{\infty}\left(\mathbb{B}_{n}\right)\right)$ if and only if $S=T_{g}+F$ with 
$g \in H^{\infty}\left(\mathbb{B}_{n}\right)$ and $\operatorname{rank}(F)<\infty$. Giving up the special structure of $H^{2}\left(\mathbb{B}_{n}\right)$, at least the following can be said.

Theorem 3.5. Let $T \in B(H)^{n}$ be a spherical isometry with $\sigma_{p}(T)=\emptyset$. Given a bounded linear operator $S \in B(H)$ such that $[S, A]$ is of finite rank for every $A \in \mathcal{A}_{T}$, then $S=X+F$ with $X \in \mathcal{T}(T)$ and a finite-rank operator $F \in B(H)$.

Proof. By the remarks preceding Proposition 3.2, the scalar-valued spectral measure $\mu$ associated with $T$ is continuous. According to Aleksandrov's approximation theorem (Proposition 2.1) there exists a weak* zero sequence of $\mu$-inner functions $\left(\theta_{k}\right)_{k \geq 1}$ in $H^{\infty}(\mu)$. By Lemma 1.1, the sequence $J_{k}=\gamma_{T}\left(\theta_{k}\right)(k \geq 1)$ is a weak* zero sequence of isometries in $\mathcal{A}_{T}$. Passing to a subsequence if necessary, we may assume that the bounded sequence $\left(J_{k}^{*} S J_{k}\right)_{k \geq 1}$ converges to an operator $X \in B(H)$ with respect to the weak* topology. By Lemma 3.4(b) (applied to $\mathcal{A}=\mathcal{A}_{T}$ ) there is a constant $M>0$ such that $\operatorname{rank}\left(\left[S, J_{k}\right]\right) \leq M$ for every $k \geq 1$. Now applying part (a) of the cited lemma we deduce that the operator

$$
F=S-X=\mathrm{w}^{*}-\lim _{k}\left(S-J_{k}^{*} S J_{k}\right)=\mathrm{w}^{*}-\lim _{k} J_{k}^{*}\left(J_{k} S-S J_{k}\right)
$$

is at most of rank $M$. It remains to check that $X$ is a Toeplitz operator. In order to verify the criterion established in Proposition 3.1 we fix an arbitrary isometry $V \in \mathcal{A}_{T}$ and write

$$
V^{*} J_{k}^{*} S J_{k} V=J_{k}^{*} V^{*} S V J_{k}=J_{k}^{*} V^{*}(V S+S V-V S) J_{k}=J_{k}^{*} S J_{k}+J_{k}^{*} V^{*}[S, V] J_{k} .
$$

Note that the last summand satisfies $\left|\left\langle J_{k}^{*} V^{*}[S, V] J_{k} x, y\right\rangle\right| \leq\left\|[S, V] J_{k} x\right\| \cdot\left\|J_{k} y\right\|$ for arbitrary vectors $x, y \in H$. Using the fact that the compact operator $[S, V]$ maps the weak zero sequence $\left(J_{k} x\right)_{k \geq 1}$ in $H$ to a norm zero sequence, we deduce that $J_{k}^{*} V^{*}[S, V] J_{k} \stackrel{k}{\longrightarrow} 0$ (WOT). Thus passing to WOT-limits in the above algebraic identity yields

$$
V^{*} X V=X \quad\left(V \text { being an arbitrary isometry in } \mathcal{A}_{T}\right),
$$

as desired.

C) Spherical isometries of class $C_{.0}$. We finally take a look at spherical isometries $T \in B(H)^{n}$ whose functional calculus satisfies a certain additional continuity condition. Here "functional calculus" does not refer to the dual algebra isomorphism $\gamma_{T}: H^{\infty}(\mu) \rightarrow \mathcal{A}_{T}$ but to another natural extension of the polynomial functional calculus of $T$ arising as follows. The von Neumann-type inequality

$$
\|p(T)\| \leq\|p(U)\| \leq\|p\|_{\infty, \partial \mathbb{B}_{n}} \quad(p \in \mathbb{C}[z]),
$$

which $T$ inherits from its spherical unitary extension $U$, yields a contractive $A\left(\mathbb{B}_{n}\right)$ functional calculus $\Phi_{T}: A\left(\mathbb{B}_{n}\right) \rightarrow B(H)$ for $T$. Obviously, $\Phi_{T}$ is related to $\gamma_{T}$ via the formula

$$
\Phi_{T}(f)=\gamma_{T}\left(\left[f \mid \partial \mathbb{B}_{n}\right]_{\mu}\right) \quad\left(f \in A\left(\mathbb{B}_{n}\right)\right) .
$$

A spherical isometry $T$ is said to be of class $C_{.0}$ if $\Phi_{T}$ satisfies the additional continuity assumption that

$$
\Phi_{T}\left(f_{k}\right)^{*} \stackrel{k \rightarrow \infty}{\longrightarrow} 0
$$

whenever $\left(f_{k}\right)$ is a Montel sequence, that is, a bounded sequence in $A\left(\mathbb{B}_{n}\right)$ such that $f_{k} \mid \mathbb{B}_{n}$ is a pointwise zero sequence. It is well known that a sequence $\left(f_{k}\right)_{k}$ in $A\left(\mathbb{B}_{n}\right)$ is of this type precisely when $\left(\left[f_{k} \mid \partial \mathbb{B}_{n}\right]\right)_{k}$ is a weak ${ }^{*}$ zero sequence in $H^{\infty}(\sigma)$ 
or equivalently in $L^{\infty}(\sigma)$, where $\sigma$ denotes the normalized surface measure on the unit sphere $\partial \mathbb{B}_{n}$. For details, the reader is referred to [10] or [8].

If $T$ is of class $C_{.0}$, then one easily deduces that the map $\Phi_{T}: A\left(\mathbb{B}_{n}\right) \rightarrow B(H)$ extends to a weak* continuous contractive homomorphism $H^{\infty}\left(\mathbb{B}_{n}\right) \rightarrow B(H)$, again denoted by $\Phi_{T}$. In this case, the composition

$$
r_{\mu}: H^{\infty}\left(\mathbb{B}_{n}\right) \stackrel{\Phi_{T}}{\longrightarrow} \mathcal{A}_{T} \stackrel{\gamma_{T}^{-1}}{\longrightarrow} H^{\infty}(\mu)
$$

yields an abstract boundary-value map, that is, a contractive and weak* continuous homomorphism mapping each polynomial $p \in \mathbb{C}[z]$ to its boundary-value equivalence class $\left[p \mid \partial \mathbb{B}_{n}\right]_{\mu}$ in $L^{\infty}(\mu)$. A measure $\nu \in M^{+}\left(\partial \mathbb{B}_{n}\right)$ possessing such a weak* continuous and contractive boundary-value map $r_{\nu}: H^{\infty}\left(\mathbb{B}_{n}\right) \rightarrow H^{\infty}(\nu)$ is called a Henkin measure. It is called faithful if $r_{\nu}$ is isometric and hence a dual algebra isomorphism. It is well known that the surface measure $\sigma$ on $\partial \mathbb{B}_{n}$ is a faithful Henkin measure.

As a final remark, we state that, given two Henkin measures $\eta \ll \nu$ in $M^{+}\left(\partial \mathbb{B}_{n}\right)$, the boundary homomorphisms of $\eta$ and $\nu$ are related to each other via

$$
r_{\eta}^{\nu} \circ r_{\nu}=r_{\eta}
$$

(to be checked on polynomials), where $r_{\eta}^{\nu}$ denotes the canonical weak* continuous contraction

$$
r_{\eta}^{\nu}: L^{\infty}(\nu) \rightarrow L^{\infty}(\eta), \quad[f]_{\nu} \mapsto[f]_{\eta} .
$$

Lemma 3.6. Let $T \in B(H)^{n}$ be a spherical isometry of class $C_{.0}$. Then there exists a sequence $\left(J_{k}\right)_{k \geq 1}$ of isometries in $\mathcal{A}_{T}$ satisfying

$$
J_{k}^{*} \stackrel{k \rightarrow \infty}{\longrightarrow} 0 \quad(\mathrm{SOT}) .
$$

Proof. Let $\mu \in M^{+}\left(\partial \mathbb{B}_{n}\right)$ be the scalar-valued spectral measure associated with $T$, which, by the considerations above, is a Henkin measure and thus continuous (Lemma 2.2.3 in [8]). By the approximation theorem of Aleksandrov stated as Proposition 2.1 above, there exists a sequence of $(\mu+\sigma)$-inner functions

$$
\left(\theta_{k}\right)_{k \geq 1} \text { in } I_{\mu+\sigma} \subset H^{\infty}(\mu+\sigma) \text { with } \theta_{k} \stackrel{w^{*}}{\longrightarrow} 0 \text { in } L^{\infty}(\mu+\sigma) .
$$

Since for all $f \in H^{\infty}\left(\mathbb{B}_{n}\right)$, the estimate

$$
\|f\|_{\infty, \mathbb{B}_{n}}=\left\|r_{\sigma}(f)\right\|_{\infty, \sigma}=\left\|r_{\sigma}^{\mu+\sigma}\left(r_{\mu+\sigma}(f)\right)\right\|_{\infty, \sigma} \leq\left\|r_{\mu+\sigma}(f)\right\|_{\infty, \mu+\sigma}
$$

holds, the measure $\mu+\sigma$ is a faithful Henkin measure, and so we are able to define the desired sequence of operators as

$$
J_{k}=\Phi_{T}\left(r_{\mu+\sigma}^{-1}\left(\theta_{k}\right)\right) \quad(k \geq 1) .
$$

Indeed, $\left(J_{k}^{*}\right)_{k \geq 1}$ is an SOT zero sequence in view of the $C .0$-property of $T$ and the weak* continuity of $r_{\mu+\sigma}^{-1}$. In order to check that $J_{k}$ is an isometry for $k \geq 1$, we write

$$
J_{k}=\Phi_{T}\left(r_{\mu+\sigma}^{-1}\left(\theta_{k}\right)\right)=\gamma_{T}\left(r_{\mu}\left(r_{\mu+\sigma}^{-1}\left(\theta_{k}\right)\right)\right)=\gamma_{T}\left(r_{\mu}^{\mu+\sigma}\left(\theta_{k}\right)\right)
$$

and observe that $\left|r_{\mu}^{\mu+\sigma}\left(\theta_{k}\right)\right|=1 \mu$-almost everywhere, since $\left|\theta_{k}\right|=1(\mu+\sigma)$-almost everywhere. By Lemma 1.1, the proof is complete.

Proposition 3.7. A spherical isometry $T \in B(H)^{n}$ is of class $C_{.0}$ if and only if it is completely non-unitary. 
Proof. By Corollary 2.4 in [10, every completely non-unitary spherical isometry is of class $C_{\text {.0. }}$.

To prove the opposite direction, fix a spherical isometry of class $C_{.0}$ and assume that $M \subset H$ is a reducing subspace for $T$ such that $N=T \mid M \in B(M)^{n}$ is a spherical unitary. In order to show that $M$ must be the zero space, first note that the identity $\Phi_{T}(p)^{*} \mid M=\left(\Phi_{T}(p) \mid M\right)^{*}=\Phi_{N}(p)^{*}$, trivially valid for polynomials $p \in \mathbb{C}[z]$, extends by continuity to all functions $p \in A\left(\mathbb{B}_{n}\right)$. Thus $N$ is of class $C_{._{0}}$.

Consider the isomorphism of von Neumann algebras $\Psi_{N}: L^{\infty}(\nu) \rightarrow W^{*}(N)$ associated with the spherical unitary $N$. Here, as before, we regard the scalar-valued spectral measure of $N$ as a measure $\nu \in M^{+}\left(\partial \mathbb{B}_{n}\right)$. Then $\mathcal{A}_{N}=\Psi_{N}\left(H^{\infty}(\nu)\right)$; hence by Lemma 1.1 and the lemma above, there exists a sequence of isometries $J_{k}=\Psi_{N}\left(\theta_{k}\right)$ with inner functions $\theta_{k} \in H^{\infty}(\nu)$ such that $J_{k}^{*} \rightarrow 0$ (SOT) on $M$. But since in this case the isometries $J_{k}=\Psi_{N}\left(\theta_{k}\right)$ are even unitary operators, we see that

$$
\|x\|=\left\|J_{k}^{*} x\right\| \stackrel{k \rightarrow \infty}{\longrightarrow} 0 \quad(x \in M) .
$$

This proves that $M$ is the zero space, as was to be shown.

Let us finally remark that the class of spherical isometries possesses various generalizations associated with suitable subsets of $\mathbb{C}^{n}$ which are "far away from the ball" but still allow Aleksandrov's construction of inner functions (e.g. the class of $\partial D$-isometries over a bounded strictly pseudoconvex domain $D \subset \mathbb{C}^{n}$ considered by Athavale in [3] or the class of regular $A$-isometries introduced by the second author in [11). As expected, the results contained in Section 3 possess natural analogues in these contexts. The details will be presented elsewhere.

\section{REFERENCES}

1. A.B. Aleksandrov, Inner functions on compact spaces, Funct. Anal. Appl. 18 (1984), 87-98. MR745695 (86d:32003)

2. A. Athavale, On the intertwining of joint isometries, J. Operator Theory 23 (1990), 339-350. MR.1066811 (91i:47029)

3. A. Athavale, On the intertwining of $\partial D$-isometries, Complex Analysis and Operator Theory 2, No. 3 (2008), 417-428. MR2434460 (2009h:47013)

4. H. Bercovici, A factorization theorem with applications to invariant subspaces and the reflexivity of isometries, Math. Res. Lett. 1 (1994), 511-518. MR.1302394 (95m:47005)

5. A. Brown, P.R. Halmos, Algebraic properties of Toeplitz operators, J. reine und angewandte Mathematik 213 (1963/64), 89-102. MR0160136 (28:3350)

6. J.B. Conway, Towards a functional calculus for subnormal tuples: The minimal normal extension, Trans. Amer. Math. Soc. 326 (1991), 543-567. MR1005077 (91k:47048)

7. K.R. Davidson, On operators commuting with Toeplitz operators modulo the compact operators, J. Functional Analysis 24 (1977), 291-302. MR0454715 (56:12963)

8. M. Didas, Dual algebras generated by von Neuman $n$-tuples over strictly pseudoconvex sets, Dissertationes Math. 425 (2004). MR2067612 (2005d:47009)

9. M. Didas, Spherical isometries are reflexive, Integr. Equ. Oper. Theory 52 (2005), 599-604. MR2184607 (2006g:47009)

10. J. Eschmeier, Invariant subspaces for spherical contractions, Proc. London Math. Soc. 75 (1996), 157-176. MR:1444317 (98j:47007)

11. J. Eschmeier, On the reflexivity of multi-variable isometries, Proc. Amer. Math. Soc. 134 (2006), 1783-1789. MR2207494 (2006k:47010)

12. K. Guo, Essential commutants of analytic Toeplitz algebra and some related problems, Acta Math. Sinica 39 (1996), 300-313 [in Chinese]. MR1413350 (97i:47043) 
13. K. Guo, K. Wang, On operators which commute with analytic Toeplitz operators modulo the finite rank operators, Proc. Amer. Math. Soc. 134 (2006), 2571-2576. MR 2213734 (2007b:47074)

14. B. Prunaru, Some exact sequences for Toeplitz algebras of spherical isometries, Proc. Amer. Math. Soc. 135 (2007), 3621-3630. MR2336578 (2008g:47145)

Fachrichtung Mathematik, Universität des SaArlandes, Postfach 151150, D-66041 SaArbrüCken, Germany

E-mail address: didas@math.uni-sb.de

Fachrichtung Mathematik, Universität des SaArlandes, Postfach 151150, D-66041 SaArbrüCKen, Germany

E-mail address: eschmei@math.uni-sb.de 